\title{
Alternative configurations of quantile regression for estimating predictive uncertainty in water level forecasts for the upper Severn River: a comparison
}

\author{
P. López López ${ }^{1,2, *}$, J. S. Verkade ${ }^{2,3,4}$, A. H. Weerts ${ }^{2,5}$, and D. P. Solomatine ${ }^{1,3}$ \\ ${ }^{1}$ UNESCO-IHE Institute for Water Education, Delft, the Netherlands \\ ${ }^{2}$ Deltares, Delft, the Netherlands \\ ${ }^{3}$ Delft University of Technology, Delft, the Netherlands \\ ${ }^{4}$ Ministry of Infrastructure and the Environment, Water Management Centre of the Netherlands, \\ River Forecasting Service, Lelystad, the Netherlands \\ ${ }^{5}$ Wageningen University and Research Centre, Wageningen, the Netherlands \\ * currently at: Utrecht University (Utrecht) and Deltares (Delft), the Netherlands
}

Correspondence to: P. López López (patricia.lopez@deltares.nl)

Received: 21 March 2014 - Published in Hydrol. Earth Syst. Sci. Discuss.: 7 April 2014

Revised: 17 July 2014 - Accepted: 25 July 2014 - Published: 8 September 2014

\begin{abstract}
The present study comprises an intercomparison of different configurations of a statistical post-processor that is used to estimate predictive hydrological uncertainty. It builds on earlier work by Weerts, Winsemius and Verkade (2011; hereafter referred to as WWV2011), who used the quantile regression technique to estimate predictive hydrological uncertainty using a deterministic water level forecast as a predictor. The various configurations are designed to address two issues with the WWV2011 implementation: (i) quantile crossing, which causes non-strictly rising cumulative predictive distributions, and (ii) the use of linear quantile models to describe joint distributions that may not be strictly linear. Thus, four configurations were built: (i) a "classical" quantile regression, (ii) a configuration that implements a non-crossing quantile technique, (iii) a configuration where quantile models are built in normal space after application of the normal quantile transformation (NQT) (similar to the implementation used by WWV2011), and (iv) a configuration that builds quantile model separately on separate domains of the predictor. Using each configuration, four reforecasting series of water levels at 14 stations in the upper Severn River were established. The quality of these four series was intercompared using a set of graphical and numerical verification metrics. Intercomparison showed that reliability and sharpness vary across configurations, but in none of the
\end{abstract}

configurations do these two forecast quality aspects improve simultaneously. Further analysis shows that skills in terms of the Brier skill score, mean continuous ranked probability skill score and relative operating characteristic score is very similar across the four configurations.

\section{Introduction}

Forecasting may reduce but can never fully eliminate uncertainty about the future. Hydrological forecasts will always be subject to many sources of uncertainty, including those originating in the meteorological forecasts used as inputs to hydrological models (e.g. precipitation and temperature), and in the hydrological models themselves (e.g. model structure, model parameters and human influences). Informed decision-making may benefit from estimating the remaining uncertainties. A number of research studies suggest that enclosing predictive uncertainty estimates indeed leads to benefits for end users (Krzysztofowicz, 2001; Collier et al., 2005; Verkade and Werner, 2011; Ramos et al., 2013; Dale et al., 2014).

In the literature, various approaches to estimate predictive uncertainty have been presented. One of those is the use of meteorological ensemble forecasts, where initial 
atmospheric conditions are perturbed to yield an ensemble of atmospheric forecasts. These can be routed through a hydrological model, thus yielding an ensemble of hydrologic model forecasts which provide insight into the sensitivity of hydrological model results to various possible weather scenarios. Increasingly, hydrologic forecasting systems are including these ensemble predictions in the forecasting routines to capture the meteorological uncertainty. An overview of applications and best practices was given by Cloke and Pappenberger (2009). More recent applications include the Environment Agency's National Flood Forecasting System (NFFS) (Schellekens et al., 2011) and the US National Weather Service's Hydrologic Ensemble Forecast Service (HEFS) (Demargne et al., 2014). Note that HEFS also includes a statistical post-processor developed by Seo et al. (2006).

A second approach is statistical post-processing. Estimating predictive uncertainty through statistical post-processing techniques comprises an analysis of past, "observed" predictive uncertainty to build a model of future predictive uncertainties. It can be used as either an alternative or additional step to hydrological ensemble forecasting. In many hydrological forecasting applications, postprocessing is used in combination with deterministic forecasts (but it can also be applied to ensemble hydrologic forecasts if available; see, for example, Reggiani et al., 2009; Verkade et al., 2013). A historical record of past forecasts and their verifying observations is then used to build a model of forecast error. (Note that other configurations are possible, but this one is the most straightforward and common one.) On the assumption that this error will be similar in future cases, the error model is then applied to newly produced deterministic forecasts, thus producing an estimate of predictive hydrological uncertainty. This estimate then includes uncertainties originating in both the atmospheric forecasts as well as those in the numerical simulation of streamflow generation and routing processes. Post-processing assumes a stationary relation between the predictors and the predictors. It follows that both the forecasts and the observations used for calibration have to be stationary. Also, ideally the calibration record is sufficiently long as to include events that are (relatively) extreme. The reason for this is that the relationship between forecast and observations at extreme events may be different from the relationship in non-extreme hydrological regimes. If the assumption of stationarity cannot be met, or if the calibration record is short, the quality of the post-processed forecasts is likely to be reduced. Several hydrologic post-processors have been described in the scientific literature, including the Hydrological Uncertainty Processor (HUP; Krzysztofowicz and Kelly, 2000), Bayesian Model Averaging (BMA; Raftery et al., 2005), the Model Conditional Processor (MCP; Todini, 2008), UNcertainty Estimation based on local Errors and Clustering (UNEEC; Solomatine and Shrestha, 2009), the Hydrologic Model Output Statistics (HMOS; Regonda et al.,
2013) and quantile regression (QR; Weerts et al., 2011). The present paper focuses on the latter technique.

Quantile regression (QR; Koenker and Bassett Jr., 1978; Koenker and Hallock, 2001; Koenker, 2005) aims to describe a full probability distribution of the variable of interest (the predictand), conditional on one or more predictors. Contrary to some of the other post-processors (such as HUP or BMA), QR requires few prior assumptions about the characterization of the model error. While it was originally developed for applications in the economic sciences, it has since been introduced into environmental modelling and climate change impact assessment (e.g. Bremnes, 2004; Nielsen et al., 2006). The technique has been applied in various research studies as a post-processing technique to estimate predictive hydrological uncertainty, including those described by Solomatine and Shrestha (2009), Weerts et al. (2011), Verkade and Werner (2011), and Roscoe et al. (2012). In each of these applications, the quantiles of distribution of the model error are estimated using single valued water level or discharge forecasts as predictors.

Weerts et al. (2011; hereinafter referred to as WwV2011) describe an implementation of QR for the Environment Agency in the United Kingdom. The Historic Forecast Performance Tool (HFPT; Sene et al., 2009) makes use of QR to estimate a predictive distribution of future water levels using the deterministic water level forecast as a predictor. The WwV2011 configuration of QR includes a transformation into Gaussian space using the normal quantile transformation (NQT) (Krzysztofowicz and Kelly, 2000; Montanari and Brath, 2004; Bogner and Pappenberger, 2011). In QR, the quantiles are estimated one at a time. Potentially, these quantiles cross, thus yielding implausible predictive distributions. The quantile crossing problem was addressed by omitting the domain of the predictor where the crossing occurred from the QR procedure and instead, in that domain, imposed a prior assumed distribution of the predictand.

The results of the WWV2011 analysis were verified for reliability and showed to be satisfactory. However, this verification was unconditional in the sense that only the full available sample of paired (probabilistic) forecasts and observation was assessed for reliability. When the HFPT was further tested (Vaughan, 2012), it was noticed that the probabilistic forecasts did not perform equally well in high flow conditions. One of the contributions of the present paper consists of a conditional analysis of forecast skill. Forecast skill is assessed for progressively higher flood levels, in terms of commonly used verification metrics and skill scores. These include Brier's probability score, the continuous ranked probability score and corresponding skill scores as well as the relative operating characteristic score.

The configuration of QR in WWV2011 included two elements that, in the present paper, are explored in additional detail. These steps are (i) the technique for avoiding crossing quantiles and (ii) the derivation of regression quantiles in normal space using the normal quantile transformation (NQT). 
In WWV2011, quantile crossing was avoided by manually imposing a distribution of the predictand in the domain of the predictor where crossing occurred. Since designing and implementing that particular configuration, an alternative technique for estimating non-crossing quantile regression curves has emerged (Bondell et al., 2010). As the latter technique requires less manual interference by the modellers, the present paper investigates whether implementation thereof yields estimates of predictive uncertainty that are of equal or higher quality.

In WWV2011, QR was applied using first-degree polynomials, i.e. describing the distribution of the predictand as a linear function of the predictor. This, of course, assumes that the joint distribution of predictor and predictand can be described in linear fashion. To facilitate this, both marginal distributions (of forecasts and of observations) were transformed into normal or Gaussian domain using the NQT. The joint distribution was subsequently described in normal space using linear regression quantiles, and then back-transformed into original space. The resulting regression quantiles are then no longer linear. While this procedure yielded satisfactory results, there is no requirement on the part of QR of either the marginal or joint distributions to have marginal or joint normal distribution. Also, the transformation, and especially the back-transformation, impose additional assumptions on the marginal distributions and can thus be problematic. Hence a justified question is whether this transformation to and from normal space actually yields better results. In the present paper, this is tested by comparing multiple configurations of QR: derivation of regression quantiles in original space and in normal space. As an additional step, a piecewise linear configuration is tested, where the domain of the predictor is split into several, mutually exclusive and collectively exhaustive domains, on each of which the regression quantiles are calibrated.

The objective of this work is to thoroughly verify uncertainty estimates using the implementation of $\mathrm{QR}$ that was used by WWV2011, and to intercompare forecast quality and skill in various, differing configurations of QR. The configurations are (i) "classical" $\mathrm{QR}$, (ii) QR constrained by a requirement that quantiles do not cross, (iii) $\mathrm{QR}$ derived on time series that have been transformed into the normal domain (similar to WWV2011 QR configurations, with the exception of how the quantile crossing problem is addressed), and (iv) a piecewise linear derivation of $\mathrm{QR}$ models. A priori, it is expected that imposing a non-crossing requirement yields results that are at least as good as those of the "classical" implementation of $\mathrm{QR}$, and that derivation in normal space and piecewise linear derivation each constitute a further improvement in quality and skill compared to derivation in original space.

The novel aspects and new contributions of this work include the thorough verification of an earlier implementation of $\mathrm{QR}$, the application of the non-crossing $\mathrm{QR}$ to this particular case study and the exploration of techniques for

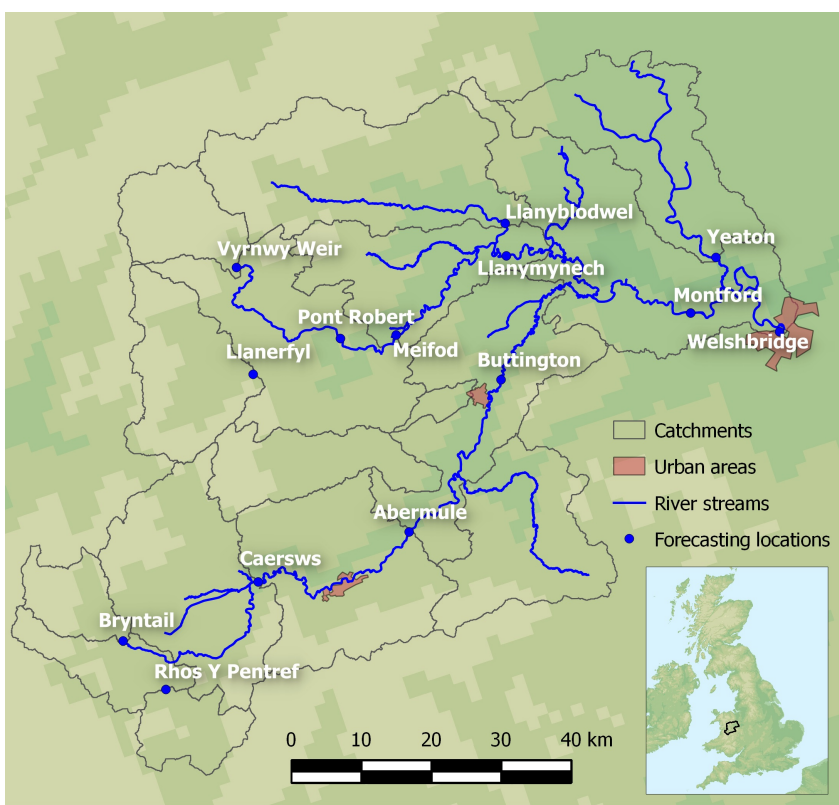

Figure 1. The upper Severn Basin including the 14 forecasting locations used in the present study. Note that the smallest river streams are not shown in the stream network. (The digital elevation model is made available by the European Environment Agency on a Creative Commons Attribution License; http://www.eea.europa.eu/ data-and-maps/data/digital-elevation-model-of-europe.)

ensuring that joint distributions can be described using linear QR models.

This paper first describes the approach, materials and methods, including the study basin, the hindcasting process, the analysed QR configurations and the verification process. Subsequently, results and analysis are presented. The paper ends with conclusions and discussion.

\section{Approach, materials and methods}

The present study consists of an experiment in which verification results of four differently configured post-processors (each based on the quantile regression technique) are intercompared. By the varying configurations, two potential issues are addressed: quantile crossing and possible nonlinearity of the joint distribution of predictor and predictand.

\subsection{Study basin: upper Severn River}

The upper Severn Basin (Fig. 1) serves as the study basin for the present study. Its predominantly hilly catchment extends from the Welsh Hills at Plynlimon to the gauge at Welshbridge in Shrewsbury and is approximately $2284 \mathrm{~km}^{2}$ large. Lake Vyrnwy (Vyrnwy River) and Llyn Clywedog (Clywedog River) are two reservoirs located in the headwaters of the catchment. The upper Severn includes rock formations classified as non-aquifers as well as loamy soils characterized 
Table 1. Hydrometeorological and topographical information of analysed catchments at upper Severn River (adapted from EA, 2013 and Marsh and Hannaford, 2008).

\begin{tabular}{llcccccc}
\hline Station name & River & $\begin{array}{c}\text { Basin area } \\
\left(\mathrm{km}^{2}\right)\end{array}$ & $\begin{array}{c}\text { Elevation } \\
(\mathrm{m} \mathrm{AOD})\end{array}$ & $\begin{array}{c}\text { Mean } \\
\text { annual rainfall } \\
(\mathrm{mm})\end{array}$ & $\begin{array}{c}\text { Mean flow } \\
\left(\mathrm{m}^{3} \mathrm{~s}^{-1}\right)\end{array}$ & $\begin{array}{c}\text { Highest river } \\
\text { level recorded } \\
(\mathrm{m})\end{array}$ & $\begin{array}{c}\text { Basin } \\
\text { lag time } \\
(\mathrm{h})\end{array}$ \\
\hline Caersws & Severn & - & 119 & - & - & 3.69 & $8-10$ \\
Abermule & Severn & 580 & 83 & 1291 & 14.58 & 5.26 & $13-17$ \\
Buttington & Severn & - & 62 & - & - & 5.5 & $8-10$ \\
Montford & Severn & 2025 & 52 & 1184 & 43.3 & 6.96 & $10-15$ \\
Welshbridge & Severn & 2025 & 47 & - & - & 5.25 & $15-20$ \\
Vyrnwy Weir & Vyrnwy & 94.3 & 226.34 & 1951 & 4.24 & 1.8 & $2-5$ \\
Pont Robert & Vyrnwy & 675 & 100 & - & - & 3.07 & $5-9$ \\
Meifod & Vyrnwy & 675 & 81 & - & - & 3.67 & $7-10$ \\
Llanymynech & Vyrnwy & 778 & 62 & 1358 & 21.08 & 5.19 & $3-6$ \\
Bryntail & Clywedog & 49 & 212.05 & 2026 & 2.4 & 1.61 & $2-4$ \\
Rhos Y Pentref & Dulas & 52.7 & 178.49 & 1313 & 1.45 & 2.42 & $1-3$ \\
Llanerfyl & Banwy & - & 151 & - & - & 3.5 & $3-5$ \\
Llanyblodwel & Tanat & 229 & 77.28 & 1267 & 6.58 & 2.68 & $7-10$ \\
Yeaton & Perry & 108.8 & 61.18 & 767 & 1.6 & 1.13 & $15-20$ \\
\hline
\end{tabular}

by their high water retention capacity. Annual precipitation varies, with topography from 700 to $2500 \mathrm{~mm}$ (EA, 2009). Flooding occurs relatively frequently, with major floods occurring in autumn 2000, February 2002, 2004, summer 2007, fall 2012 as well as at the time of writing this manuscript, early 2014. To manage flood risk, the UK Environment Agency developed the River Severn Catchment Flood Management Plan in 2009. Flood risk management is supported by the Midlands Flood Forecasting System (MFFS), which is based on the Delft-FEWS forecast production system (Werner et al., 2013). The upper Severn configuration in MFFS consists of a sequence of numerical models for modelling of rainfall-runoff (MCRM; Bailey and Dobson, 1981), hydrological routing (DODO; Wallingford, 1994) and hydrodynamical routing (ISIS; Wallingford, 1997) processes as well as an internal MCRM error correction procedure based on the Autoregressive Moving Average (ARMA) technique. The input data for MFFS consists of Real Time Spatial (RTS) data (observed water level data, rain gauge data, air temperature and catchment average rainfall data), Radar Actuals, Radar Forecast, and Numerical Weather Prediction data. This input data is provided by the UK Meteorological Office.

The uncertainty models are used to estimate predictive uncertainty at 14 hydrological stations on the upper Severn River, each having different catchments characteristics. Figure 1 shows a map with the forecasting locations and their basins. Table 1 summarizes some key hydrological data.

\subsection{Hindcasting process}

The uncertainty models (Sect. 2.3) are derived using a joint historical record of observations and forecasts. The latter is acquired through the process of reforecasting or hindcasting.
For this, a stand-alone version of the forecast production system MFFS is used. Prior to every forecast, the models are run in historical mode over the previous period to produce an estimate of internal states (groundwater level, soil moisture deficit, snow water equivalent, snow density, etc). In this historical mode, models are forced with observed precipitation, evapotranspiration and temperature. The system is subsequently run in forecast mode twice daily, with forecast issue times of 08:00 and 20:00 UTC, with a maximum lead time of $48 \mathrm{~h}$. The selected reforecasting period is from $1 \mathrm{Jan}-$ uary 2006 through 7 March 2013. Of this period, the period up to 6 March 2007 is used to "spin up" the models. The remaining 6 years are used for the calibration and validation of the uncertainty models.

\subsection{Uncertainty models}

In the present study, predictive uncertainty is modelled using quantile regression. The basic configuration is simple, and identical across all cases: the predictive distribution of future observed water levels is modelled as a series of quantiles, each estimated as a linear function of a single predictor which is the deterministic water level forecast. Four different configurations are intercompared. Configuration $0(\mathrm{QR} 0)$ constitutes the most straightforward case, where QR is applied "as is", i.e. in its most basic form, in which no attempt is made to avoid crossing quantiles and no transformation or piecewise derivation is applied. Configuration 1 (QR1) addresses the problem of the crossing quantiles using the technique proposed by Bondell et al. (2010). If the quantile crossing problem does not occur, this technique provides the same estimates as in the base scenario. Because of this, it is also applied to the remaining configurations. In some cases, the 
Table 2. QR configurations used in the present study

\begin{tabular}{ll}
\hline Identifier & Description \\
\hline QR0 & $\begin{array}{l}\text { Classical quantile regression - base scenario } \\
\text { Quantile regression constrained by a } \\
\text { non-crossing quantiles restriction }\end{array}$ \\
QR2 & $\begin{array}{l}\text { Quantile regression, constrained by a } \\
\text { non-crossing quantiles restriction, on the } \\
\text { transformed data into normal domain through } \\
\text { normal quantile transformation (NQT) } \\
\\
\text { Piecewise linear derivation of quantile } \\
\text { regression, constrained by a non-crossing } \\
\text { quantiles restriction }\end{array}$ \\
\hline
\end{tabular}

joint distribution of forecasts and observations is not best modelled using linear quantile regression models across the full domain of the predictor. However, by applying a transformation or by modelling sub-domains of the predictor, linear models may be used nonetheless. This is what is done in configurations $2(\mathrm{QR} 2)$ and $3(\mathrm{QR} 3)$, respectively. The configurations are each described in detail in the following four sub-sections; for reference, they are also listed in Table 2. As the non-crossing quantiles are applied to configurations 2 through 4 , the comparison in the present paper is effectively between these three latter configurations (QR1, QR2 and QR3).

The joint distribution of forecasts and their verifying observations is based on the UK Environment Agency archives of water level observations and on the forecasts from the hindcasting procedure. The available record is crossvalidated through a leave-one-year-out cross-validation analysis. From the 6 years' worth of forecasts that are available for calibration and validation, 5 are used for model calibration and the single remaining year is used for model validation. Subsequently, another year is chosen for validation and the calibration period then comprises the remaining 5 years. This is repeated until all 6 years have been used for validation.

Uncertainty models are developed for each combination of lead time and location separately. While the forecasts have a maximum lead time of $48 \mathrm{~h}$ with 1-hour intervals, the QR models are derived on a limited number of lead times, namely for $1 \mathrm{~h}$ lead time and then 3 through $48 \mathrm{~h}$ lead time with 3-hour increments. The leave-one-year-out cross-validation procedure yields approximately 3760 observation-forecast pairs for every combination of lead time and location.

\subsubsection{QR0: quantile regression}

Quantile regression (Koenker and Bassett Jr., 1978; Koenker and Hallock, 2001; Koenker, 2005) is a regression technique for estimating the quantiles of a conditional distribution. As the sought relations are conditional quantiles rather than conditional means, quantile regression is robust with regards to outliers. Quantile regression does not make any prior assumptions regarding the shape of the distribution; in that sense, it is a non-parametric technique. It is, however, highly parametric in the sense that for every quantile of interest, parameters need to be estimated.

In the present work, quantile regression is used to estimate lead time $n$-specific conditional distributions of water level,

$\phi_{n}=\left\{H_{n, \tau_{1}}, H_{n, \tau_{2}}, \ldots, H_{n, \tau_{T}}\right\}$,

where $T$ is the number of quantiles $\tau(\tau \in[0,1])$ considered. If $T$ is sufficiently large and the quantiles $\tau$ jointly cover the domain $[0,1]$ sufficiently well, we consider $\phi_{n}$ to be a continuous distribution. Here, $T=25$ and $\tau \in\{0.02,0.06, \ldots, 0.98\}$,

$\phi_{n}=\left\{H_{n, \tau=.02}, H_{n, \tau=.06}, \ldots, H_{n, \tau=.98}\right\}$.

We assume that (cf. WWV2011), separately for every lead time $n$ considered, for every quantile $\tau$ there is a linear relationship between the water level forecast $S$ and its verifying observation $H$ :

$H_{n, \tau}=a_{n, \tau} S_{n}+b_{n, \tau}$

where $a_{n, \tau}$ and $b_{n, \tau}$ are the slope and intercept from the linear regression. Quantile regression allows for finding the parameters $a_{n, \tau}$ and $b_{n, \tau}$ of this linear regression by minimising, through a process of linear programming, the sum of residuals:

$\min \sum_{j=1}^{J} \rho_{n, \tau}\left(h_{n, j}-\left(a_{n, \tau} s_{n, j}+b_{n, \tau}\right)\right)$,

where $h_{j}$ and $s_{j}$ are the $j$ th paired samples from a total of $J$ samples, $a_{n, \tau}$ and $b_{n, \tau}$ the regression parameters from the linear regression between water level forecast and observation, respectively, and $\rho$ is the quantile regression function for the $\tau$ th quantile:

$\rho_{n, \tau}\left(\varepsilon_{n, j}\right)=\left\{\begin{array}{lll}(\tau-1) \varepsilon_{n, j} & \text { if } \quad \varepsilon_{n, j} \leq 0 \\ \tau \varepsilon_{n, j} & \text { if } \quad \varepsilon_{n, j}>0 .\end{array}\right.$

The quantile regression function (Eq. 5) is applied for the residual $\left(\varepsilon_{n, j}\right)$, which is defined as the difference between the observation $\left(h_{n, j}\right)$ and the linear QR estimate $\left(a_{n, \tau} s_{n, j}+\right.$ $\left.b_{n, \tau}\right)$ for the selected quantile, $\tau$ and the specific lead time, $n$. By varying the value of $\tau$, the technique allows for describing the entire conditional distribution of the dependent variable $H$.

In the present work, solving Eq. (4) was done using the quantreg package (Koenker, 2013) in the R software environment (R Core Team, 2013). Figures 2, 3 and 4 give a graphical overview of the resulting quantiles. These plots are discussed in the Results and Analysis section. 


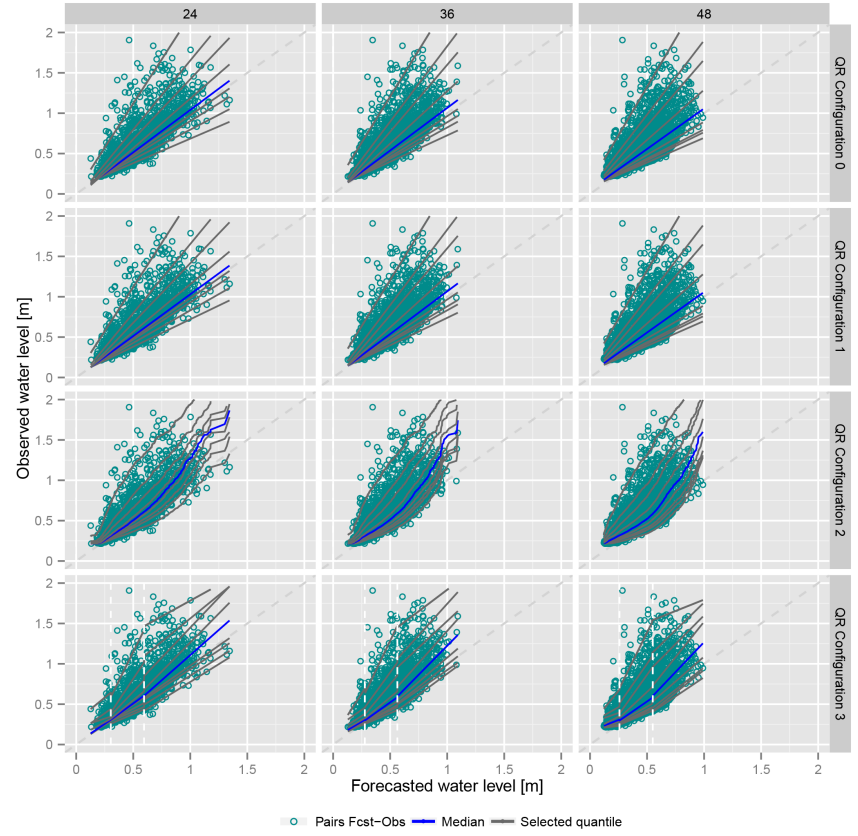

Figure 2. Quantile regression models for Llanyblodwel. Rows show the four different configurations; columns show different lead times.

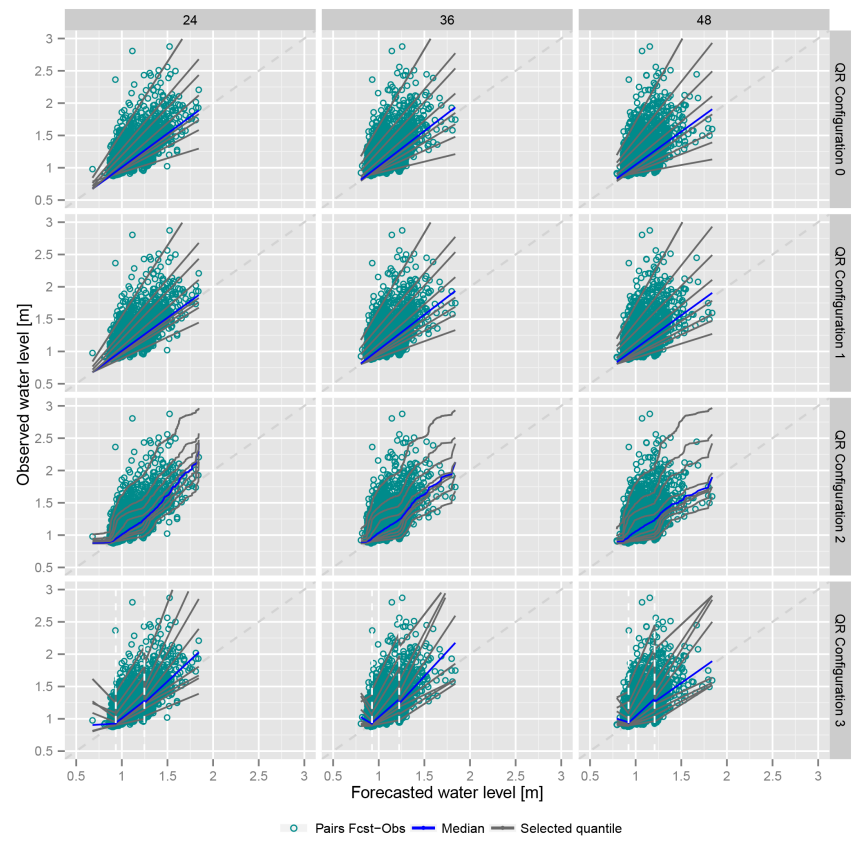

Figure 3. Quantile regression models for Pont Robert. Rows show the four different configurations; columns show different lead times.

\subsubsection{QR1: non-crossing quantile regression}

A potential problem with using $\mathrm{QR}$ for the derivation of multiple conditional quantiles is that quantiles may cross, yielding predictive distributions that are not, as a function of increasing quantiles, monotonously increasing. WWV2011

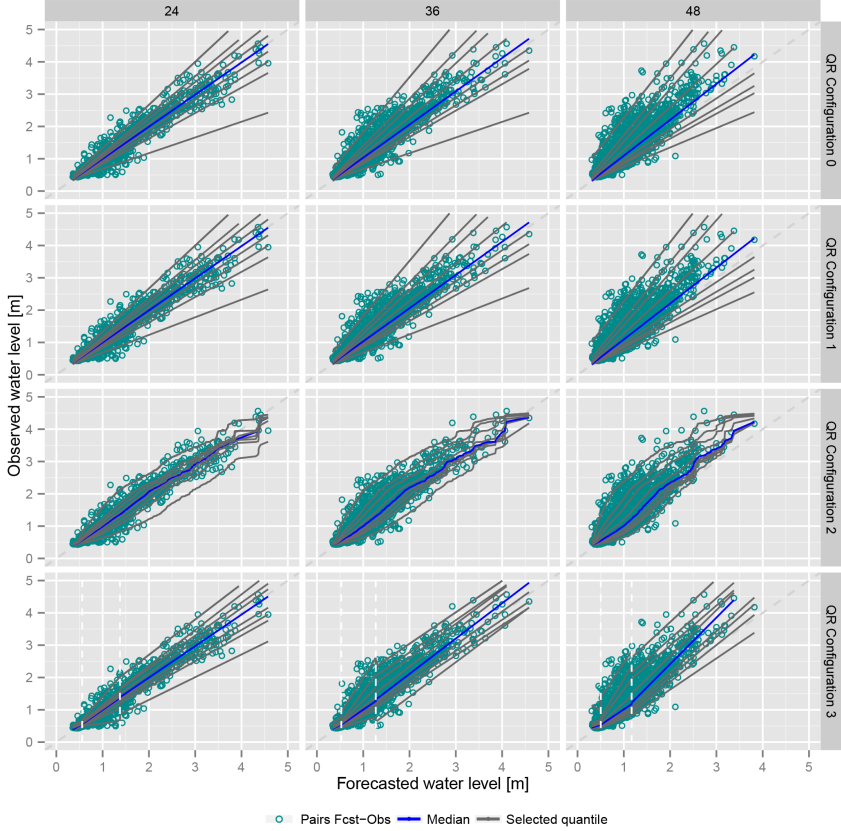

Figure 4. Quantile regression models for Welshbridge. Rows show the four different configurations; columns show different lead times.

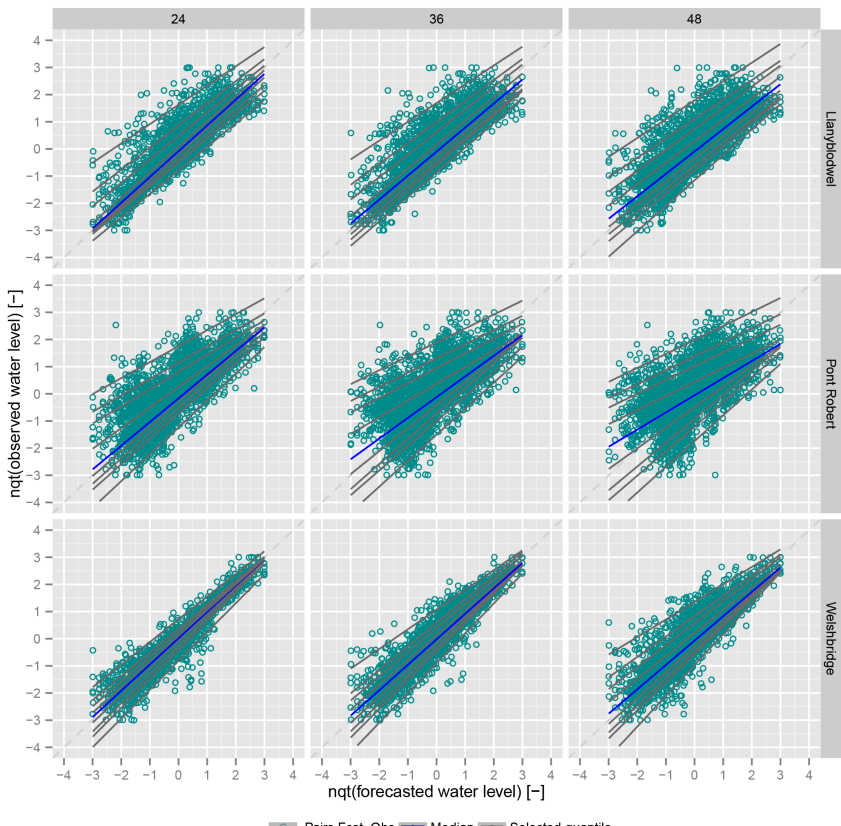

Figure 5. Quantile regression models for Llanyblodwel, Pont Robert and Welshbridge in normal space (QR2). Rows show the three different locations; columns show different lead times.

have addressed this issue by assuming a fixed error model in the domain of the predictor where there is the danger of quantiles crossing. In the present research study, the technique proposed by Bondell et al. (2010) is used. This technique imposes a non-crossing restriction on the solution of 
Eq. 4. Without this restriction, the solution to the proposed optimization problem is identical to that of classical quantile regression, i.e. to the models derived using QR0. For a more detailed description of the non-crossing quantiles technique, the reader is referred to Bondell et al. (2010). The technique is freely available online (NCSU Statistics, 2010) and is coded in the statistical computing language $\mathrm{R}$ ( $\mathrm{R}$ Core Team, 2013).

\subsubsection{QR2: quantile regression in normal space}

In this configuration, time series of water level observations and water level forecasts are first transformed into the normal domain. This results in time series that have marginal normal distribution. Subsequently, quantile regression models are calibrated using the non-crossing quantiles technique. After the derivation of QR models, the variables are backtransformed into original space. The rationale for using the transformation is that the joint distribution of transformed time series appears to be more linear, and can thus be better described by linear conditional quantiles.

The normal quantile transformation (NQT) is a quantile mapping or cdf-matching technique that matches the (empirical or modelled) cdf of the marginal distributions with a standard normal cdf. Here, the empirical cdf of the marginal distributions is used. Thus, the variables are mapped to a standard normal distribution:

$$
\begin{aligned}
H_{\mathrm{nqt}} & =\mathrm{Q}^{-1}(\mathrm{~F}(H)) \\
S_{\mathrm{nqt}, n} & =\mathrm{Q}^{-1}\left(\mathrm{~F}\left(S_{n}\right)\right),
\end{aligned}
$$

where $\mathrm{F}(\cdot)$ is the Weibull plotting position of the data point considered. The equivalent of Eq. (3) then becomes

$H_{\mathrm{nqt}, n, \tau}=a_{n, \tau} S_{\mathrm{nqt}, n}+b_{n, \tau}$,

which is solved by minimizing the sum of residuals:

$\min \sum_{j=1}^{J} \rho_{n, \tau}\left(h_{\mathrm{nqt}, n, j}-\left(a_{n, \tau} s_{\mathrm{nqt}, n, j}+b_{n, \tau}\right)\right)$.

After the analysis in normal space, the variables are backtransformed to original space using a reversed procedure:

$$
\begin{aligned}
& H=\mathrm{Q}\left(\mathrm{F}\left(H_{\mathrm{nqt}}\right)\right) \\
& S_{n}=\mathrm{Q}\left(\mathrm{F}\left(S_{\mathrm{nqt}, n}\right)\right) .
\end{aligned}
$$

Back-transformation is problematic if the quantiles of interest lie outside of the range of the empirical distribution of the untransformed variable in original space. In those cases, assumptions will have to be made on the shape of the tails of the distribution (see Bogner, $\mathrm{K}$ and Pappenberger for a more elaborate discussion). Some authors have chosen to parameterize the distribution of the untransformed variable and use those statistical models for the back-transformation (see, for example, Krzysztofowicz and Kelly, 2000). In the present study, this matter is treated through a linear extrapolation on a number of points in the tails of the distribution which was the solution chosen by Montanari (2005) and by WwV2011.

\subsubsection{QR3: piecewise linear quantile regression}

In an effort to try and use linear quantile models to describe a joint distribution that may be slightly non-linear in nature, Van Steenbergen et al. (2012) applied linear models to partial domains of the predictor. They found the resulting distributions to be both more reliable and sharper. Multiple, mutually exclusive and collectively exhaustive domains were identified based on a visual inspection of the data and taking into account the requirement that each sub-group will have to contain a sufficiently sized data sample. As this selection more or less coincided with two splits at the 20th and 80th percentile, three sub-domains were defined, comprising 20 , 60 and $20 \%$ of the data respectively.

\subsection{Verification strategy}

To understand and intercompare the performance of different QR configurations, an extensive verification of forecast quality was carried out. The post-processing procedure separated calibration from validation hence the verification can be considered to be independent. The old(-ish) adage has it that probabilistic forecasts should strive for sharpness subject to reliability (Gneiting et al., 2005): an improvement in sharpness at the expense of reliability is not desirable. In addition, decision-makers may be interested in event discrimination skills for specific flood thresholds, for example. Forecasts were therefore assessed for reliability, sharpness and event discrimination, and a number of metrics were calculated.

These verification metrics include the Brier score (BS), the mean continuous ranked probability score (CRPS) and area beneath the relative operating characteristic (ROCA). Reliability was assessed using reliability diagrams that plot the relative frequency of occurrence of an event versus the predicted probability of event occurrence. Proximity to the $1: 1$ diagonal, where observed frequency equals predicted probability, indicates higher reliability. Sharpness was explored by determining the width of the centred $80 \%$ interval of the predictive distributions; the full sample of these widths is shown by means of an empirical cumulative distribution function (ecdf). The Brier score (Brier, 1950) is defined as the mean squared error of a probabilistic forecast of a binary event. The mean CRPS (Brown, 1974; Matheson and Winkler, 1976) is a measure of the squared probabilistic error in the forecasts across all possible discrete events. The area beneath the relative operating characteristic is a measure of the forecasts' ability to discriminate between the exceedance and non-exceedance of a threshold, for example a flood threshold. A detailed description of these measures with their mathematical formulation can be found in Appendix A. 


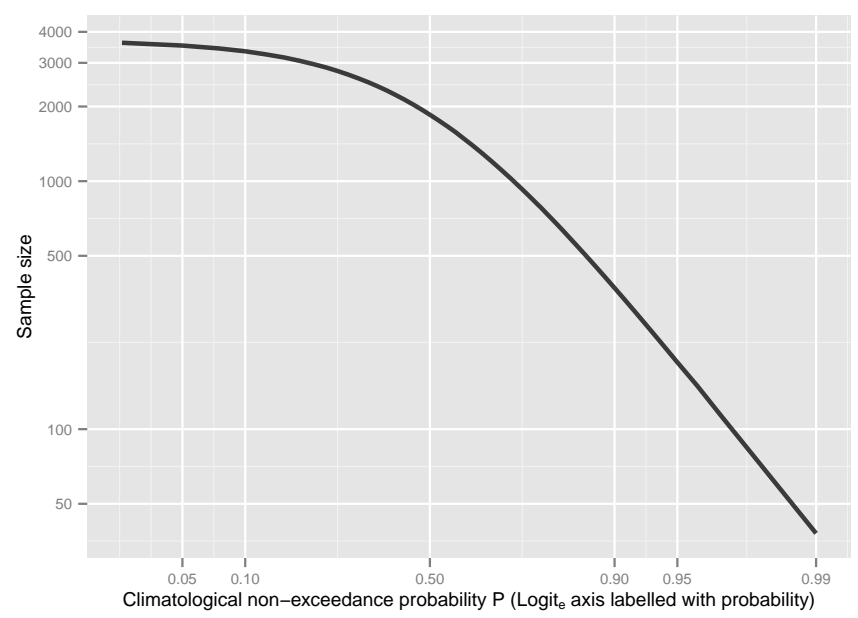

Figure 6. Sample size as a function of the climatological probability of non-exceedance $P$.

To allow for comparison across different locations, BS, CRPS and ROCA are expressed as skill, thus becoming the Brier skill score (BSS), continuous ranked probability skill score (CRPSS) and the relative operating characteristic score (ROCS):

skill $=\frac{\text { score }- \text { score }_{\text {ref }}}{\text { score }_{\text {perfect }}-\text { score }_{\text {ref }}}$,

where score is the score of the system considered, score $_{\text {ref }}$ is the score of a reference system and score perfect $_{\text {is the highest }}$ possible score. Skill scores range from $-\infty$ to 1 . The highest possible value is 1 . If skill $=0$, the system's score is as good as that of the reference system. If skill $<0$ then the system's score is less than that of the reference system. In the case of BSS and CRPSS, the reference score comprises that of the sample unconditional climatology; in the case of ROCS, the reference score is the ROCA associated with an unskilled forecast which states that the probability of an event occurrence is equal to the probability of non-event occurrence.

As the post-processor is intended to be used in flood forecasting, forecast skill is not only assessed for the full available sample of forecast observation pairs, but also for subsets of high and extreme events. These sub-sets are defined by the climatological probability of non-exceedance $P$ of the observation. For example, $P=0.95$ denotes the sub-sample of forecast, observation pairs where the observation falls in the top $5 \%$ of observations. Increasing the value of $P$ from 0 (i.e. the full available sample) to a value close to 1 thus gives an indication of forecast performance for high events.

By construction, sample size for the computation of every verification metric varies with the climatological probability of non-exceedance $P$ considered (Fig. 6). Increasing the value of $P$ means lower sample size. Sampling uncertainties of the verification metrics were explored by bootstrapping. The stationary block bootstrapping technique was applied. This method constructs resample blocks of observations to form a pseudo-time series, so that the statistic of interest may be recalculated based on the resampled data set (Politis and Romano, 1994). The minimum sample size was set to 50 and the number of bootstrap samples to use in computing the confidence intervals was set to 1000 . The applied resampling method estimates the sampling distribution of each verification score. Here, the 5th and 95th percentiles of those distributions are shown. These thus constitute the centred $90 \%$ confidence intervals. Verification metrics were calculated using the Ensemble Verification System (Brown et al., 2010).

\section{Results and analysis}

Results were produced for each of the 14 locations listed in Table 1 and all of the lead times were considered. For practical reasons, the present section includes results for a limited number of lead times and locations only: 24, 36 and 48-hour lead times at Llanyblodwel, Pont Robert and Welshbridge, respectively. This combination thus comprises forecasting locations with varying sizes of contributing area. Pont Robert is located upstream, Llanyblodwel somewhere in the middle, and Welshbridge at the very outlet of the upper Severn Basin.

\subsection{Uncertainty models}

Uncertainty models for the three locations are shown in Figs. 2, 3 and 4. All scatter plots show observed water levels on the vertical axis versus water level forecasts on the horizontal axis. Each of the figures consists of a matrix of multiple panels, with rows showing the four configurations considered and columns showing various lead times. Note that across configurations, the scattered pairs are identical. On the scatter plots, a summary of the estimated uncertainty models is superimposed, consisting of a selection of quantiles only: $\tau \in\{0.01,0.05,0.1,0.25,0.5,0.75,0.9,0.95,0.99\}$. Note that these quantiles were derived for plotting purposes only, and do not necessarily coincide with the quantiles derived for verification. In the analysis, a more elaborate set of quantile is used. The latter quantiles are derived using a leave-oneyear-out procedure (see Sect. 2.3 for details), whereas this was not the case for the example quantiles in Figs. 2 through 5. However, the derived models do not differ markedly. In the plots, the QR-estimated quantiles are shown in grey, with the exception of the median quantile which is shown in blue.

From Figs. 2, 3 and 4, a few general observations can be made. All scatter plots show that there is an obvious correlation of forecasted and observed water levels, although in none of the combinations of location and lead time, all forecasts are equal to the observations. With the spread of the forecast, the observation pairs increase with increasing lead time. At zero lead time, the error correction technique ensures that modelled (i.e. simulated or forecasted) water levels are equal to the water level observation, hence at issue time there is no forecasting uncertainty. With increasing lead 


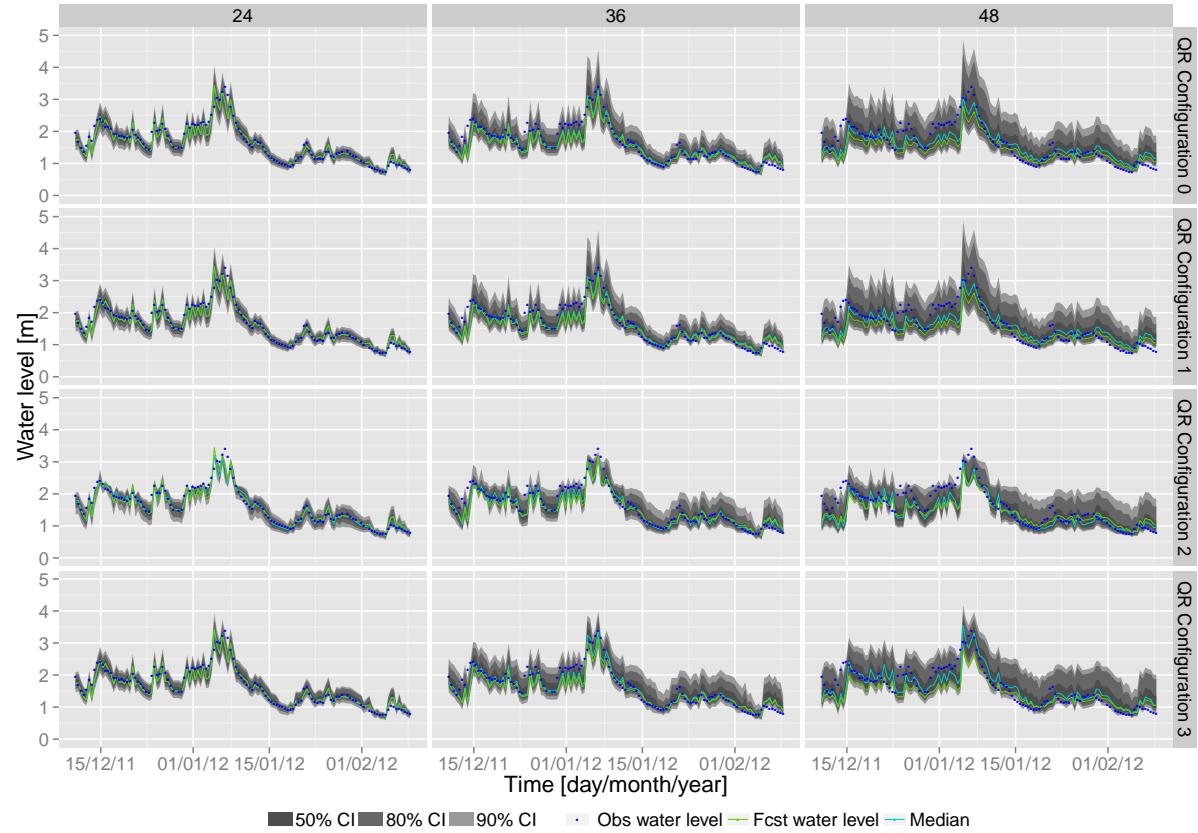

Figure 7. Hydrographs of late 2011 and early 2012 events at Welshbridge.

time, this uncertainty increases. The location with the largest lag time (Welshbridge) shows a spread that is more concentrated around the 1:1 diagonal than the other locations that have smaller contributing areas and shorter lag times. The location and slope of the quantiles show that in most cases, spread is modelled to be very small at low predicted values of the forecast, and increases with increasing value of the forecast.

The figures show how the uncertainty models, each based on a different configuration of quantile regression, differ from one another. Configurations 0 and 1 appear to be very similar. They differ only in those instances where the former configuration would lead to quantile crossing but are otherwise identical, which was indeed anticipated. Configurations 2 (derived using NQT) and 3 (piecewise linear approach) are quite different from the first two configurations, but not dissimilar to one another. In these configurations, the quantiles are not a linear function of the water level forecast, that is, not along the full domain. Note that this non-linearity constituted the very reason why these configurations were included in the analysis. Both models often - but not always - show a very small spread at the lowest water level forecasts, followed by an increasing spread. At high water level forecasts, however, spread no longer increases and sometimes decreases. This means that sharpness of the resulting probability forecasts then no longer reduces with increasing values of the water level prediction; sometimes it even increases.

Figure 5 gives some additional background to the QR2 scenario and shows the estimated quantiles in normal space, i.e. prior to back-transformation to original space. Similar to the other configurations, the estimated quantiles are linear.
The strong non-linearity that is shown in Figs. 2 through 4 is a result of the back-transformation from normal to original space.

From the pairs and the models, we can see that at both Llanyblodwel and Pont Robert, the deterministic forecast has a tendency towards underforecasting, i.e. to underestimate future water levels. This underforecasting is corrected for by the uncertainty models, that thus include a bias correction by resulting in a median forecast that is higher than the deterministic forecast. The joint forecast observation distribution for Welshbridge shows that there is much less obvious underforecasting, or overforecasting for that matter.

\subsection{Hydrographs}

Hydrographs are shown in Fig. 7 at Welshbridge for a flood event that took place late 2011 and early 2012. The multiplot panel is composed by three columns representing three different lead times; 24, 36 and 48-hour, and four rows for each of the four QR configurations. Each of these plots shows time in the horizontal axis, approximately 3 months, and water level in the vertical axis. Deterministic forecast water level (green line), observations (blue dots), median quantile (light blue) and centred 50, 80 and $90 \%$ confidence intervals are included (in shades of grey). Across the configurations for a particular lead time, water level observations and deterministic forecasts are identical.

From the plots, a number of observations can be made, each consistent with what was to be expected based on the QR models. Uncertainty increases with lead time, as is shown by the widest intervals at the highest lead times, and vice 


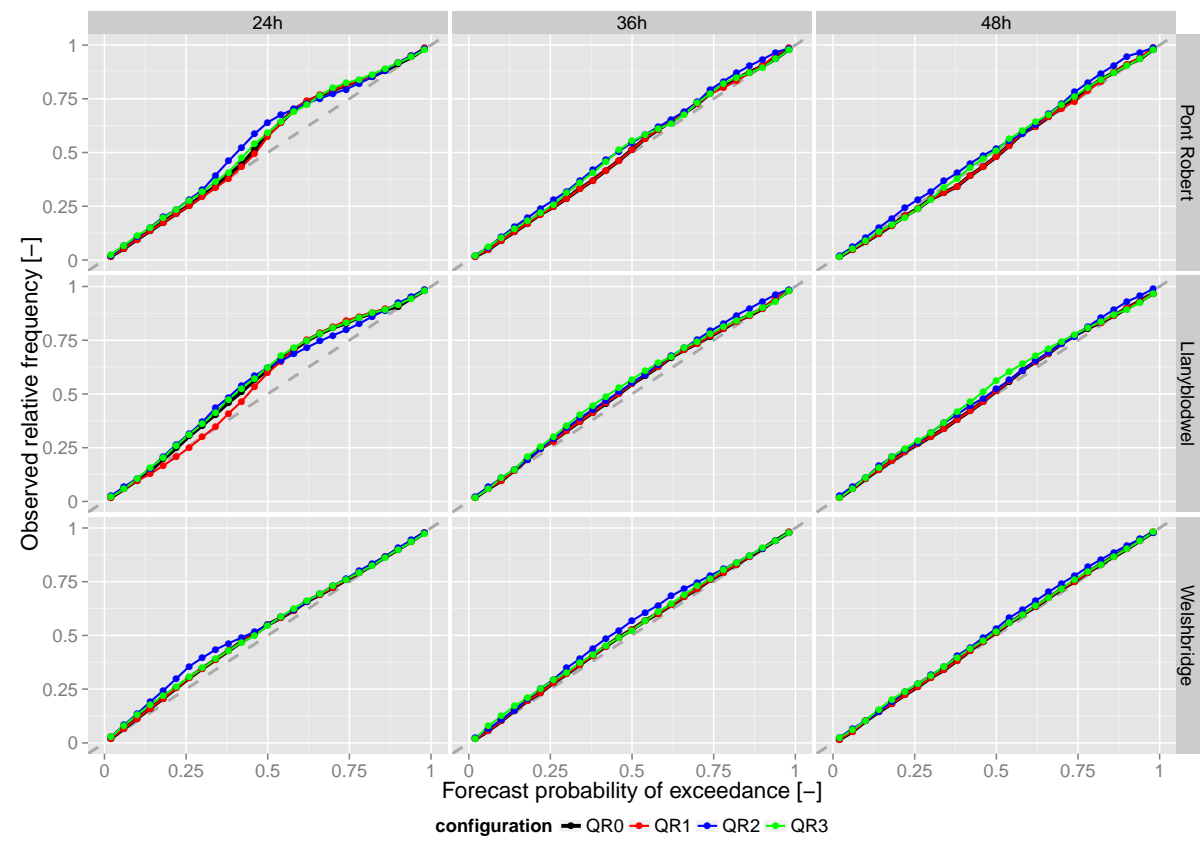

Figure 8. Full sample reliability plots.

versa. The deterministic forecast tends to underestimate water level observations. With increasing lead time, underforecasting increases. At a 48-hour lead time for high water levels, the deterministic forecast provides a higher underestimation than for low and medium water levels, which is consistent with QR models shown in Fig. 4.

The probabilistic forecasts resulting from configurations 0 and 1 are quite similar to one another. They both show the highest uncertainty at higher deterministic water level forecasts. Configuration 2 does not show this behaviour; at higher deterministic forecasts, probabilistic forecasts are sharper. Again, this is consistent with the QR model plots in Fig. 4. Configuration 3 results in forecasts whose width in the top $20 \%$ of forecasts varies only slightly (at 24-hour lead time) or almost not at all (at 36 and 48-hour lead times) with the value of the predictor.

From a visual inspection, it appears that the median quantile obtained with the four QR configurations improves the deterministic forecast. QR configurations 0 and 1 provide a median quantile with a minor improvement. Differences between the median quantile of QR configuration 2 and the deterministic forecast are the lowest ones. QR configuration 3 median quantile reproduces water level observations with the highest accuracy, including high, medium and low values.

\subsection{Verification}

\subsubsection{Reliability and sharpness}

Figures 8 and 9 show reliability diagrams for the full data sample and for the forecasts whose verifying observation falls in the top $10 \%$ of observations $(P=0.90)$, respectively. When looking at the full available sample, the diagrams show reasonably high reliability: most plotting points are very near or on the $1: 1$ diagonal. With a 24 -hour lead time, there was some underforecasting, but this is no longer the case as the longer lead times show.

At $P=0.90$, forecasts are considerably less reliable. At all locations and at all lead times, there is considerable underforecasting at all but the tails of the predictive distributions. This overforecasting is more pronounced for the smaller basins, and vice versa. Forecasts from QR0 and QR1 are equally (un-)reliable. When comparing these to forecasts from $\mathrm{QR} 2$ and $\mathrm{QR} 3$, there is no configuration that yields more, or less, reliable forecasts across all cases. QR3 forecasts are nearly always among the least unreliable forecasts, although in many cases this is a shared position with varying other configurations.

Figures 10 and 11 show the distribution of width of the centred $90 \%$ predictive intervals for the full available sample $(P=0)$ and the top $10 \%$ of observations only $(P=0.90)$, respectively. The figures show that sharpness reduces with increasing lead time as well as with increasing basin lag time. Intercomparison of sharpness between the different cases shows that for the full sample (Fig. 10) there is little if any difference between the four configurations, and virtually none between QR0 and QR1. Forecasts for events that are more extreme $(P=0.90)$ show larger differences. Again, QR0 and QR1 yield forecasts of more or less equal width, but there are some differences between these configurations and QR2 and QR3. These differences increase with 


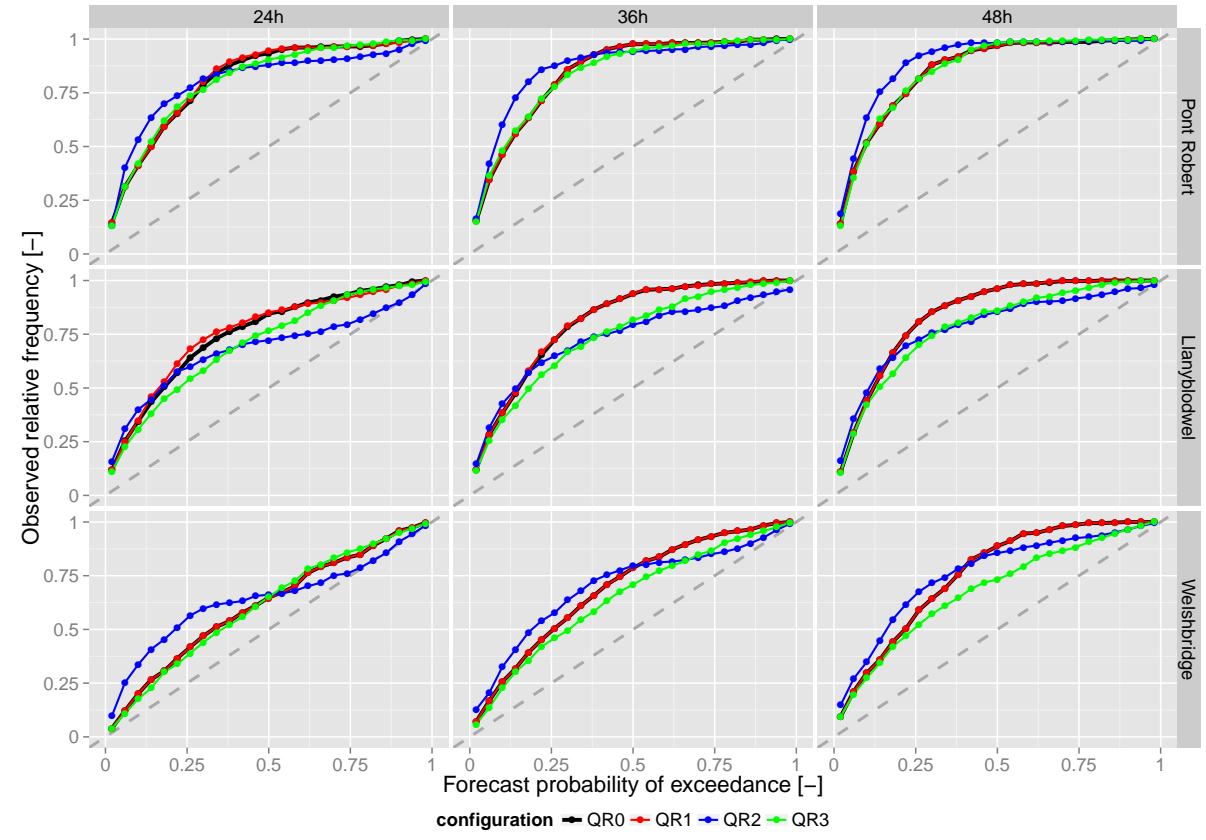

Figure 9. Reliability plots for the forecasts associated with the top $10 \%$ observations $(P=0.90)$.

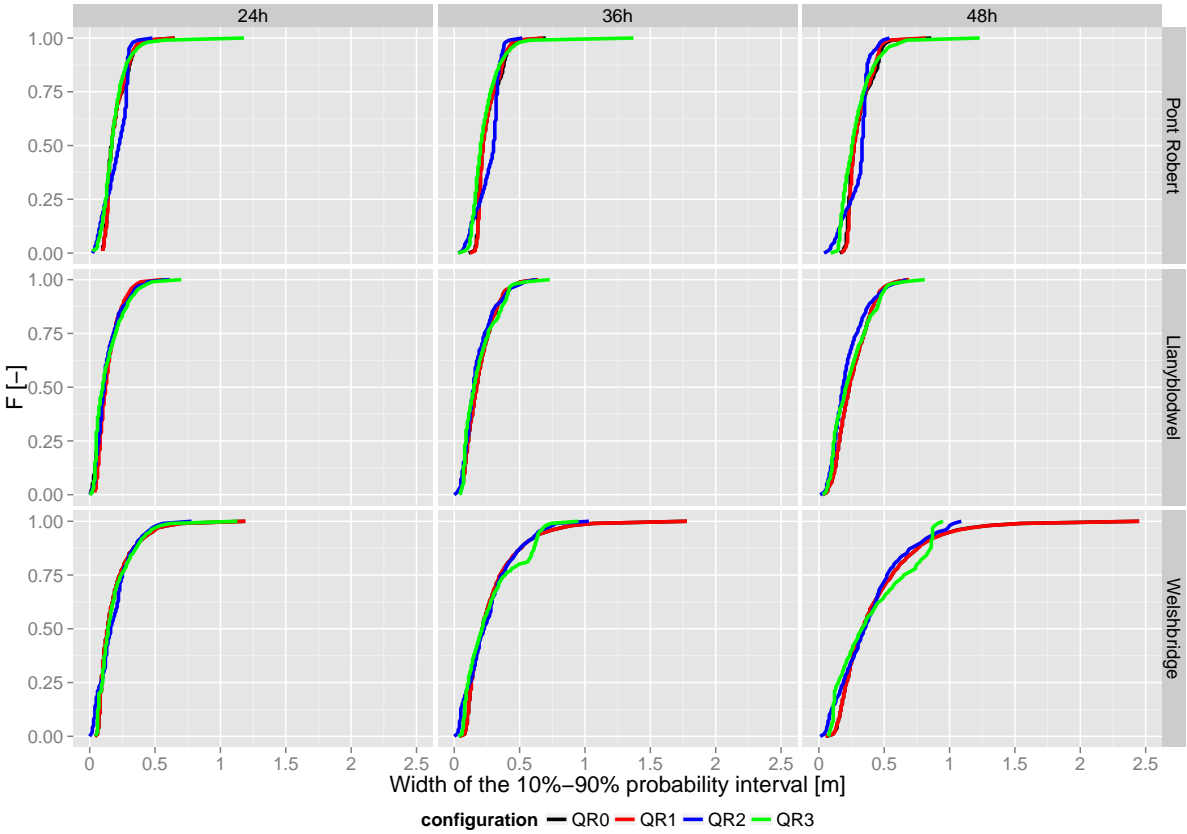

Figure 10. Empirical cumulative distribution function of the centred $80 \%$ confidence interval of the predictive distributions.

increasing lead time and increasing basin lag time. At Welshbridge, QR2 yields the sharpest forecasts, followed by QR3.

Unconditionally, both sharpness and reliability are more or less similar across the four configurations. At $P=0.90$, however, some forecasts are sharper than others but at the expense of reliability. On balance, usefulness of these forecasts may be equal. The trade-off between probability of detection and probability of false detection can be seen as a measure of this; the derived ROCS is analysed in the next section.

\subsubsection{Skill scores}

Figures 12, 13 and 14 present the skill scores computed for probabilistic forecast verification. These plots show BSS, CRPSS and ROCS (vertical axes; each score on a new row) 


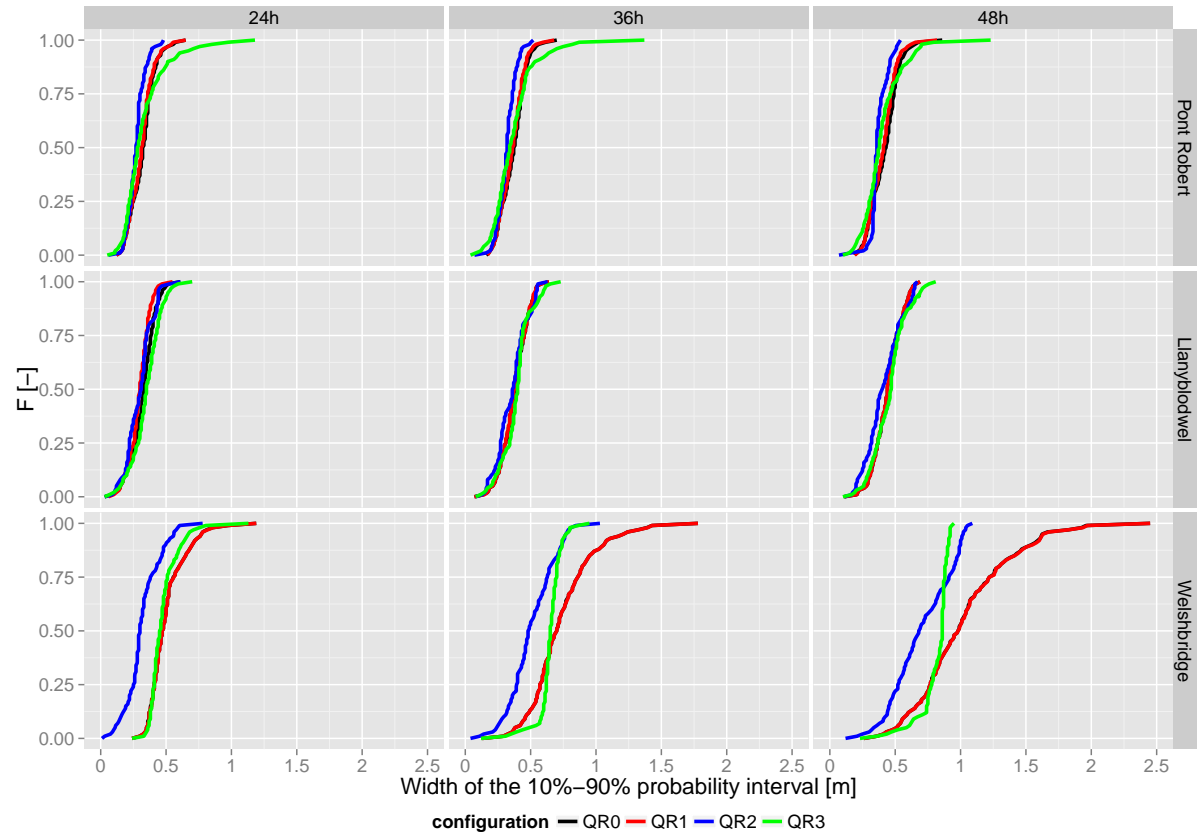

Figure 11. Empirical cumulative distribution function of the centred $80 \%$ confidence interval of the predictive distributions associated with the top $10 \%$ observations.

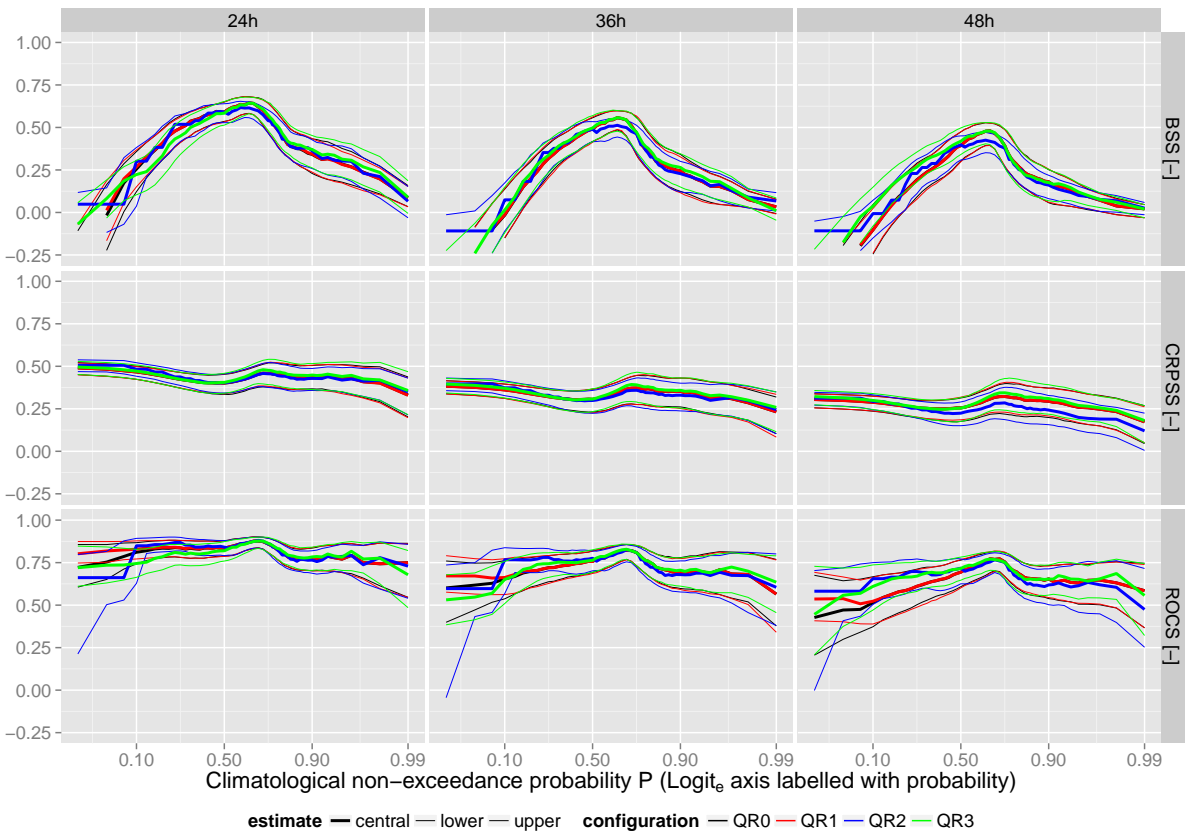

Figure 12. Verification results for water level forecasts at Pont Robert station (5-9 h lag time). In the rows, three different metrics are shown; from top to bottom these are the Brier skill score (BSS), the mean continuous ranked probability skill score (CRPSS) and the relative operative characteristic score (ROCS). Columns show various lead times: 24,36 and $48 \mathrm{~h}$.

versus the magnitude of the verifying observation, as a function of the observation which is expressed by its climatological probability of non-exceedance $P$ (horizontal axes) for various lead times (columns). In each of the plots, results are shown for four QR configurations considered. To give an indication of the uncertainty in the estimation of metrics, median as well as 10 and $90 \%$ estimates are shown.

From the figures, some general observations can be made. First of all, skills are mostly positive, with the exception of BSS and ROCS at the tails of $P$. Furthermore, skills 


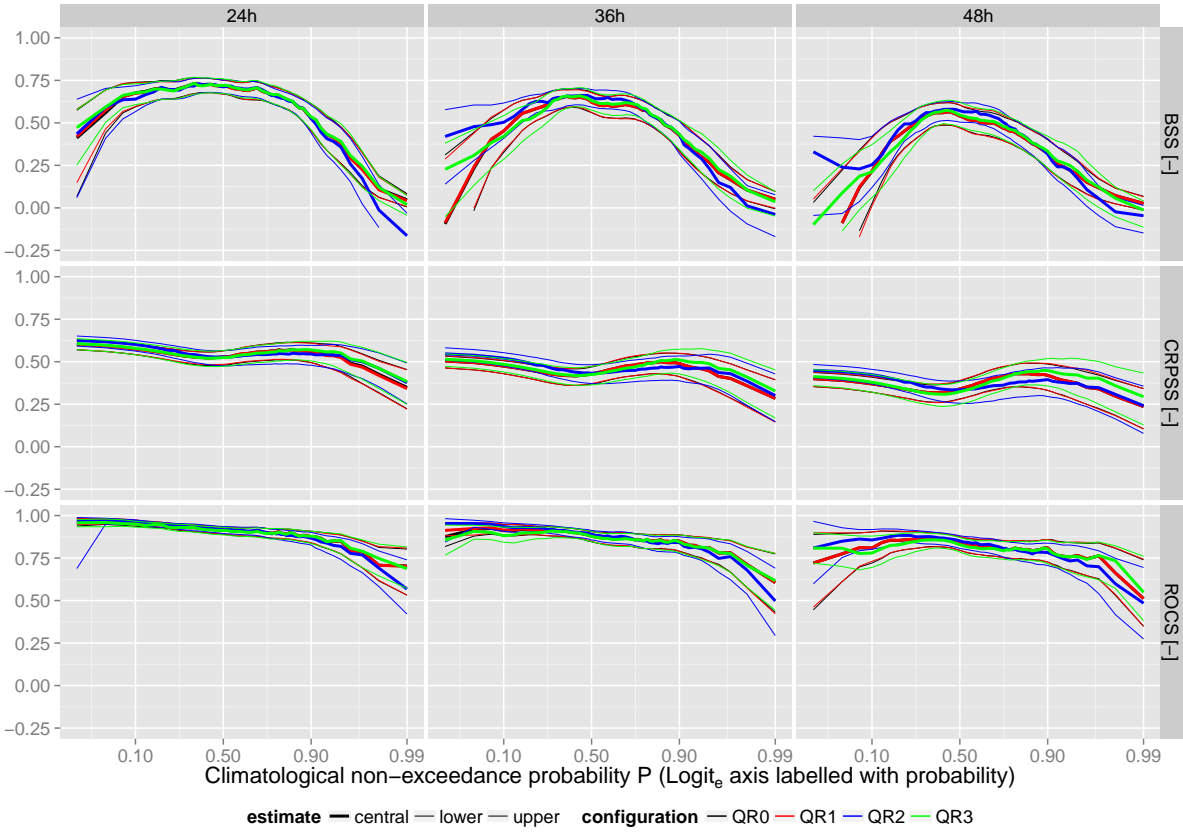

Figure 13. Verification results for water level forecasts at Llanyblodwel station (7-10 h lag time). In the rows, three different metrics are shown; from top to bottom these are the Brier skill score (BSS), the mean continuous ranked probability skill score (CRPSS) and the relative operative characteristic score (ROCS). Columns show various lead times: 24,36 and $48 \mathrm{~h}$.

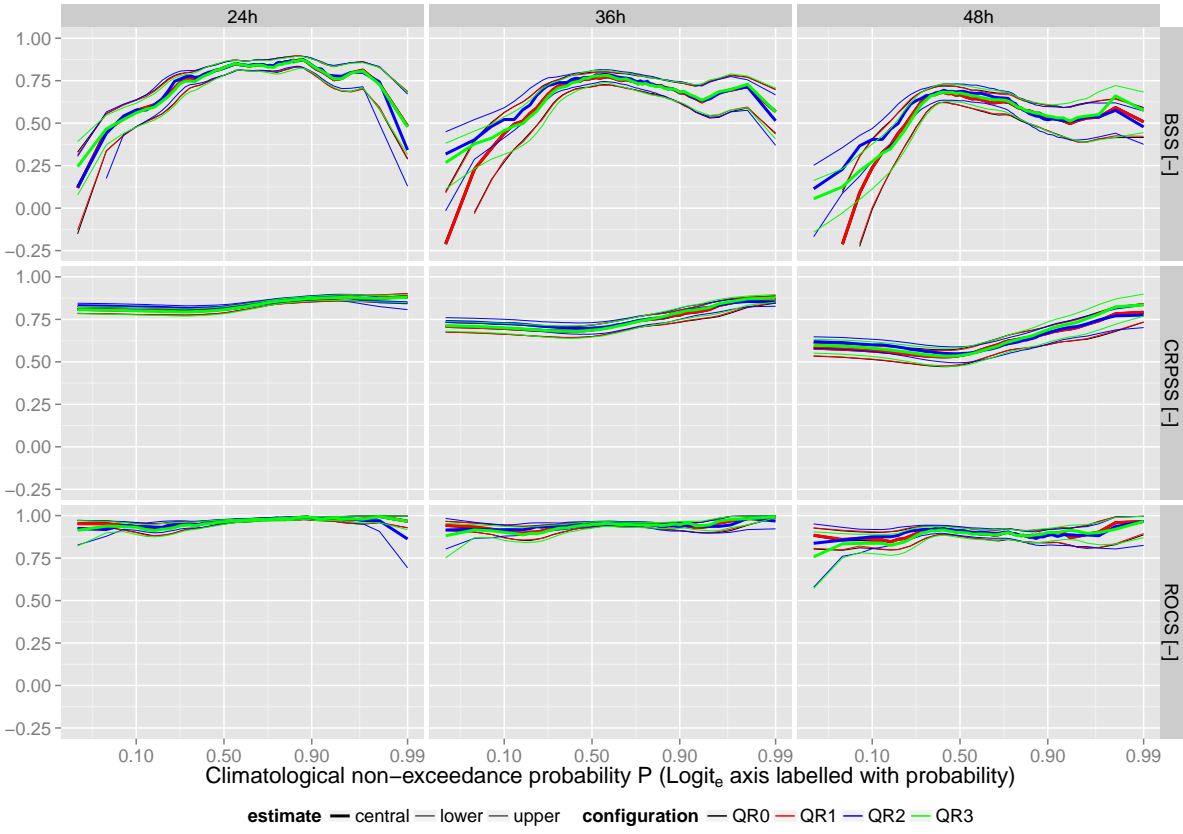

Figure 14. Verification results for water level forecasts at Welshbridge station (5-9h lag time). In the rows, three different metrics are shown; from top to bottom these are the Brier skill score (BSS), the mean continuous ranked probability skill score (CRPSS) and the relative operative characteristic score (ROCS). Columns show various lead times: 24,36 and $48 \mathrm{~h}$.

deteriorate with increasing lead time, increase with increasing basin size and vary with the observation. Many of the plotted results are very similar in that the distribution of verification metrics is very similar - both in terms of the median as well as the confidence bounds shown - across all lead times (columns) and values of $P$ (horizontal axes). As the distributions are approximations - the verification pairs used are not strictly independent - a formal statistical hypothesis testing procedure cannot be used. Hence the interpretation is necessarily largely subjective. 
The Brier skill score (BSS) as a function of $P$ has a concave, inverse $U$ shape curve. BSS is lowest - sometimes even negative - at the tails of $P$ and highest near median $P$. This is because BSS is calculated using event probabilities; and extreme events, whether low or high, are more difficult to correctly predict than non-extreme events. In terms of difference across the configurations: these are very limited. Only at the low tail do these become apparent, but often the differences are not significant.

Contrary to BSS and ROCS, CRPSS is a smooth, continuous measure that factors skill across all possible thresholds for each paired sample. This different formulation is reflected in its behaviour with increasing value of the observation. For short lead times, CRPSS is approximately constant. With increasing lead time, a small dip in CRPSS values is detected close to the median $P$. At nearly all lead times, the four $\mathrm{QR}$ configurations show very similar skill. The only exception is the highest lead times $(48 \mathrm{~h}$ ), in which QR configuration 3 outperforms the remaining cases.

ROCS is a binary event skill with a similar formulation to BSS. However, ROCS values do not show the same pattern as BSS. ROCS is largely constant for the whole climatological distribution of the observations, as it can be seen at Welshbridge in Fig. 11. Pont Robert (Fig. 12) and Llanyblodwel (Fig. 13) present lower skill for the top half of the observations. Forecast quality decreases with increasing lead time, as with BSS and CRPSS. No significant differences can be pointed out among the analysed QR configurations.

\section{Summary, conclusions and discussion}

The research described in this paper had two objectives: (i) to extensively verify the estimates of predictive uncertainty for upper Severn basins that were produced using the quantile regression post-processing technique as described by wwV2011; and (ii) to address two issues with the "as is" implementation of linear models of QR: (a) invalid predictive distributions due to the crossing quantile problem; and (b) the description of slightly non-linear joint distributions by a linear QR model.

The verification of forecast quality builds on the verification that was carried out in an earlier paper (Weerts et al., 2011). In the present paper, multiple metrics and skill scores are presented. Also, a 'conditional verification' was carried out, that is the verification was done for a large number of sub-sets of available data, each representative for increasingly higher events. Verification showed that, unconditionally, in terms of all skills and metrics, forecast quality is positive. However, the analysis also shows that forecast quality and skill decreases with increasing value of the event.

The two issues described above were addressed by implementing several techniques, thus arriving at four configurations of quantile regression. The problem of crossing quantiles was addressed by adopting the non-crossing quantiles technique that was proposed by Bondell et al. (2010). This resulted in near-identical sharpness, reliability and skill. From a forecaster's point of view, the technique constitutes a methodological improvement as the postprocessor will no longer produce invalid predictive distributions as a result of crossing quantiles, at no noticeable extra computational expense. The problem of linearly describing joint distributions of forecasts and observations that may not be linear in nature was addressed by two different approaches. The transformation to the normal space attempted to produce a joint distribution that is 'more linear'. The piecewise linear derivation approach constitutes dividing the data into sub-samples on which the joint distribution is linear.

The intercomparison shows that none of the four quantile regression configurations consistently outperforms the others. Sharpness and reliability may vary across configurations, but there none results in a more favourable combination of the two. In terms of BSS, CRPSS and ROCS, the four configurations yield comparable forecast quality.

Addressing the problem of the non-linearity of the joint distributions by the solutions proposed in the present paper has not resulted in higher skill. Either the data was sufficiently linear for the techniques not to be required, or the techniques have not performed to expectation. In any case, a skill improvement does not provide a rationale for derivation of quantile regression models in normal space as was done by WwV 2011.

While none of the configurations has a proven higher skill, there may be alternative reasons for choosing one over the other. If the post-processors are used in operational forecasting systems, the forecasters will have to be able to explain to an end user how predictive uncertainty was estimated. Hence more complicated configurations are less likely to be used. Also, forecasts have to be consistent with forecasters' beliefs (Murphy, 1993), hence the post-processor will have to fit with the forecasters' perceptual model of forecasting error.

Like all post-processing techniques, QR requires a long calibration and validation data set containing several extreme events. If the magnitude of the forecasted water level is outside of the calibration sample range, then any estimate of hydrological predictive uncertainty is not supported by data in that range. In an operational setting, it is important for the forecaster to be aware that this issue may surface. A suggestion to overcome this issue may be to "flag" the uncertainty estimate if it is based on extrapolation outside of the calibration range. Possibly, in those cases the uncertainty estimate can be replaced by an assumed estimate that the forecasters are comfortable with.

What would be a promising route to try and improve the skill of the estimates of predictive uncertainty that are produced by quantile regression? There are multiple possible answers here. First of all, there may be merit in adding predictors, i.e. by further conditioning forecast error on additional available variables. These could, for example, include the internal state variables of a model (dry or wet) and/or available 
observations at upstream locations. This route was taken by Solomatine and Shrestha (2009) in their UNEEC approach, and by Dogulu et al. (2014). Both compare a more complex UNEEC approach to QR and found improvement in skill. Stratification of the post-processing depending on different seasons or water level ranges could represent another alternative configuration. Both the addition of predictors as well as stratification, however, introduce additional data requirements that may not be met, and in the absence of which the quality of post-processed forecasts may be reduced. Alternative techniques may be considered; a recent article by van Andel et al. (2013) discusses various techniques in the context of the HEPEX intercomparison experiment. Another option would be to fully investigate additional configurations of the piecewise linear approach. For example, c-means or Kmeans clustering would allow for partitioning data to be used to build several regression models. All the configurations intercompared in the present work are parametric quantile regression estimations. Non-parametric or semi-parametric quantile regression approaches based on local smoothing could also be considered in future studies. For example, a comparison between parametric QR configurations presented here and the non-parametric estimation of the water level or discharge conditional distribution with copulas proposed by Smith et al. (2014), would be of interest. 


\section{Appendix A: Verification metrics}

For ease of reference, the probabilistic verification metrics used in this study are briefly explained. Further details can be found in the documentation of the Ensemble Verification System (Brown et al., 2010) as well as in reference works on forecast verification (Wilks, 2006; Jolliffe and Stephenson, 2012).

\section{A1 Reliability diagrams}

One desired property of probabilistic forecasts is that the predicted probabilities coincide with observed relative frequencies. Here, reliability diagrams are shown that separately plot for each lead time (indicator $n$ is omitted from below equations) the relative frequency of non-exceedance of the estimated quantiles $f_{\tau}$ of the predictive distribution versus the probability of non-exceedance $\tau$ :

$f_{\tau}=\frac{\sum_{j=1}^{J} I_{\tau, j}}{J}$,

where $I_{\tau, j}$ is an indicator variable

$I_{\tau, j}= \begin{cases}1 & \text { if } S_{\tau, j}<H_{j} \\ 0 & \text { if } S_{\tau, j} \geq H_{j}\end{cases}$

that is determined for all $j$ of $J$ pairs of forecasts $S$ and observations $H$.

\section{A2 Sharpness}

Sharpness is indicated by the width of the centred $80 \%$ interval of the predictive distribution:

$w_{j}=S_{\tau=0.90, j}-S_{\tau=0.10, j}$

for all $J$ forecasts. Again, sharpness is separately determined for each lead time $n$ and the lead time indicators have been omitted from the above equation. The combined record $w_{j=1,2, \ldots, J}$ is shown as an empirical cumulative distribution function.

\section{A3 Brier score and Brier skill score}

For a given binary event, such as the exceedance of a flood threshold, the (half) Brier score (BS; Brier, 1950) measures the mean square error of $J$ predicted probabilities that $Q$ exceeds $q$ :

$\mathrm{BS}=\frac{1}{J} \sum_{j=1}^{J}\left\{F_{S_{j}}(q)-F_{H_{j}}(q)\right\}^{2}$,

where $F_{S_{j}}(q)=\operatorname{Pr}\left[S_{j}>q\right]$ and $F_{H_{j}}(q)= \begin{cases}1 & \text { if } H_{j}>q \text {; } \\ 0 & \text { otherwise }\end{cases}$

The Brier skill score (BSS) is a scaled representation of forecast quality that relates the quality of a particular system
Table A1. Contingency table.

\begin{tabular}{llll}
\hline & Event observed & Event NOT observed & $\sum$ \\
\hline $\begin{array}{l}\text { Warning } \\
\text { issued }\end{array}$ & hits $h$ & false alarms $f$ & $w$ \\
\hline $\begin{array}{l}\text { Warning NOT } \\
\text { issued }\end{array}$ & $\begin{array}{l}\text { missed } \\
\text { events } m\end{array}$ & $\begin{array}{l}\text { quiets/correct } \\
\text { negatives } q\end{array}$ & $w^{\prime}$ \\
\hline$\sum$ & $o$ & $o^{\prime}$ & $N$ \\
\hline
\end{tabular}

BS to that of a perfect system $\mathrm{BS}_{\text {perfect }}$ (which is equal to 0) and to a reference system $\mathrm{BS}_{\text {ref: }}$ :

$$
\begin{aligned}
\mathrm{BSS} & =\frac{\mathrm{BS}-\mathrm{BS}_{\mathrm{ref}}}{\mathrm{BS}_{\text {perfect }}-\mathrm{BS}_{\mathrm{ref}}} \\
& =\frac{\mathrm{BS}-\mathrm{BS}_{\mathrm{ref}}}{0-\mathrm{BS}_{\mathrm{ref}}}=\frac{\mathrm{BS}_{\mathrm{ref}}-\mathrm{BS}}{\mathrm{BS}_{\mathrm{ref}}} \\
& =1-\frac{\mathrm{BS}}{\mathrm{BS}_{\mathrm{ref}}} .
\end{aligned}
$$

BSS ranges from $-\infty$ to 1 . The highest possible value is 1 . If $\mathrm{BSS}=0$, the BS is as good as that of the reference system. If BSS $<0$ then the system's Brier score is less than that of the reference system.

\section{A4 Mean continuous ranked probability score and skill score}

The continuous ranked probability score (CRPS) measures the integral square difference between the cumulative distribution function (cdf) of the forecast $\mathrm{F}_{S}(q)$, and the corresponding cdf of the observed variable $\mathrm{F}_{H}(q)$, averaged across $J$ pairs of forecasts and observations:

$\overline{\mathrm{CRPS}}=\frac{1}{J} \int_{-\infty}^{\infty}\left\{\mathrm{F}_{S}(q)-\mathrm{F}_{H}(q)\right\} \mathrm{d} q$.

The continuous ranked probability skill score (CRPSS) is a scaled representation of forecast quality that relates the quality of a particular system $\overline{\text { CRPS }}$ to that of a perfect system $\overline{\mathrm{CRPS}}_{\text {perfect }}$ (which is equal to 0 ) and to a reference system $\overline{\mathrm{CRPS}}_{\text {ref }}$ :

$$
\begin{aligned}
\mathrm{CRPSS} & =\frac{\overline{\mathrm{CRPS}}-\overline{\mathrm{CRPS}}_{\mathrm{ref}}}{\overline{\overline{\mathrm{CRPS}}}_{\text {perfect }}-\overline{\mathrm{CRPS}}_{\mathrm{ref}}} \\
& =\frac{\overline{\mathrm{CRPS}}_{\overline{\mathrm{CRPS}}} \overline{\mathrm{Cef}}}{0-\overline{\mathrm{CRPS}}_{\mathrm{ref}}}=\frac{\overline{\mathrm{CRS}}_{\mathrm{ref}}-\overline{\mathrm{CRPS}}}{\overline{\mathrm{CRPS}}_{\mathrm{ref}}} \\
& =1-\frac{\overline{\mathrm{CRPS}}_{\overline{\mathrm{CRPS}}_{\text {ref }}}}{}
\end{aligned}
$$

\section{A5 Relative operating characteristic score}

For a given binary event, such as the exceedance of a flood threshold, the relative operating characteristic (ROC; Green 
and Swets, 1966) plots the hit rate or probability of detection (PoD) versus the false alarm rate or probability of false detection (PoFD) for several probability thresholds. For each probability threshold, PoD and PoFD are calculated using the elements of a contingency table, which is valid for a single probabilistic decision rule, (a probability threshold above which the discrete event is considered to occur) and are defined as follows:

$$
\begin{aligned}
\mathrm{PoD} & =\frac{\# \text { hits }}{\# \text { observed events }}=\frac{h}{o} \\
\mathrm{PoFD} & =\frac{\# \text { false alarms }}{\# \text { events not observed }}=\frac{f}{o^{\prime}} .
\end{aligned}
$$

The ROC score is a skill score that relates the area under the curve (AUC) of the considered forecast to the AUC associated with an unskilled forecast where the probability of event occurrence and probability of event non-occurrence are equal, i.e. $50 \%$ :

$\mathrm{ROCS}=2 \times(\mathrm{AUC}-0.5)$

Acknowledgements. The authors would like to acknowledge the Environment Agency for provision of the data and use of the stand-alone NFFS system required to do the analyses described in the present manuscript. The authors would also like to thank J. Demargne, P. Smith and F. Pappenberger who helped to improve our manuscript considerably.

Edited by: F. Pappenberger

\section{References}

Bailey, R. and Dobson, C.: Forecasting for floods in the Severn catchment, J. Inst. Water Engrs Sci., 35, 168-178, 1981.

Bogner, K. and Pappenberger, F.: Multiscale error analysis, correction, and predictive uncertainty estimation in a flood forecasting system, Water Resour. Res., 47, W07524, doi:10.1029/2010WR009137, 2011.

Bogner, K. and Pappenberger, F.: Technical Note: The normal quantile transformation and its application in a flood forecasting system, Hydrol. Earth Syst. Sci., 16, 1085-1094, doi:10.5194/hess16-1085-2012, 2012.

Bondell, H. D., Reich, B. J., and Wang, H.: Noncrossing Quantile Regression curve estimation, Biometrika, 97, 825-838, doi:10.1093/biomet/asq048, 2010.

Bremnes, J. B.: Probabilistic Forecasts of Precipitation in Terms of Quantiles Using NWP Model Output, Mon. Weather Rev., 132, 338-347, doi:10.1175/15200493(2004)132<0338:PFOPIT>2.0.CO;2, 2004.

Brier, G.: Verification of forecasts expressed in terms of probability, Mon. Weather Rev., 78, 1-3, 1950.

Brown, T. A.: Admissible Scoring Systems for Continuous Distributions, ED135799, Rand Corporation, Santa Monica, California, ERIC, August 1974.
Brown, J. D., Demargne, J., Seo, D.-J., and Liu, Y.: The Ensemble Verification System (EVS): A software tool for verifying ensemble forecasts of hydrometeorological and hydrologic variables at discrete locations, Environ. Modell. Softw., 25, 854-872, 2010.

Cloke, H. and Pappenberger, F.: Ensemble flood forecasting: a review, J. Hydrol., 375, 613-626, 2009.

Collier, C., Cross, R., Khatibi, R., Levizzani, V., Solheim, I., and Todini, E.: ACTIF best practice pape $r-$ the requirements of flood forecasters for the preparation of specific types of warnings, in: ACTIF international conference on innovation advances and implementation of flood forecasting technology, Troms $\varnothing$, Norway, 17-19, 2005.

Dale, M., Wicks, J., Mylne, K., Pappenberger, F., Laeger, S., and Taylor, S.: Probabilistic flood forecasting and decision-making: an innovative risk-based approach, Nat. Hazards, 70, 159-172, 2014.

Demargne, J., Wu, L., Regonda, S., Brown, J., Lee, H., He, M., Seo, D.-J., Hartman, R., Herr, H. D., Fresch, M., Schaake, J., and Zhu, Y.: The Science of NOAA's Operational Hydrologic Ensemble Forecast Service, B. Am. Meteorol. Soc., 95, 79-98, doi:10.1175/BAMS-D-12-00081.1, 2014.

Dogulu, N., López López, P., Solomatine, D. P., Weerts, A. H., and Shrestha, D. L.: Predicting model uncertainty using quantile regression and UNEEC methods and their comparison on contrasting catchments, Hydrol. Earth Syst. Sci., submitted, 2014.

EA: Environment Agency: River levels: Midlands, available at: http://www.environment-agency.gov.uk/homeandleisure/floods/ riverlevels/, last access: 1 October 2013.

Gneiting, T., Raftery, A., Westveld, A., and Goldman, T.: Calibrated probabilistic forecasting using ensemble Model Output Statistics and minimum CRPS estimation, Mon.Weather Rev., 133, 1098$1118,2005$.

Green, D. M. and Swets, J. A.: Signal detection theory and psychophysics, John Wiley \& Sons, Inc., New York, 1966.

Jolliffe, I. T. and Stephenson, D. B.: Forecast Verification: a Practitioner's Guide in Atmospheric Science, John Wiley \& Sons, The Atrium, Southern Gate, Chichester, West Sussex, UK, 2012.

Koenker, R.: Quantile Regression, Cambridge University Press, 2005.

Koenker, R.: quantreg: Quantile Regression, R package version 5.05, available at: http://CRAN.R-project.org/package= quantreg, last access: 1 October 2013.

Koenker, R. and Bassett Jr., G.: Regression Quantiles, Econometrica, 1, 33-50, 1978.

Koenker, R. and Hallock, K.: Quantile Regression, The Journal of Economic Perspectives, 15, 143-156, 2001.

Krzysztofowicz, R.: The case for probabilistic forecasting in hydrology, J. Hydrol., 249, 2-9, 2001.

Krzysztofowicz, R. and Kelly, K.: Hydrologic Uncertainty Processor for probabilistic river stage forecasting, Water Resour. Res., 36, 3265-3277, doi:10.1029/2000WR900108, 2000.

Marsh, T. and Hannaford, J.: UK hydrometric register, Hydrological data UK series. Centre for Ecology and Hydrology, Wallingford, UK, 1-210, 2008.

Matheson, J. E. and Winkler, R. L.: Scoring rules for continuous probability distributions, Manage. Sci., 22, 1087-1096, doi:10.1287/mnsc.22.10.1087, 1976. 
Montanari, A.: Deseasonalisation of hydrological time series through the Normal Quantile Transform, J. Hydrol., 313, 274282, 2005.

Montanari, A. and Brath, A.: A stochastic approach for assessing the uncertainty of rainfall-runoff simulations, Water Resour. Res., 40, W01106, doi:10.1029/2003WR002540, 2004.

Murphy, A.: What is a good forecast? An essay on the nature of goodness in weather forecasting, Weather Forecast., 8, 281-293, 1993.

Nielsen, H. A., Madsen, H., and Nielsen, T. S.: Using Quantile Regression to extend an existing wind power forecasting system with probabilistic forecasts, Wind Energy, 9, 95-108, 2006.

Politis, D. N. and Romano, J. P.: The stationary bootstrap, J. Am. Stat. Assoc., 89, 1303-1313, 1994.

Raftery, A. E., Gneiting, T., Balabdaoui, F., Polakowski, M.: Using Bayesian model averaging to calibrate forecast ensembles, Mon. Weather Rev., 133, 1155-1174, 2005.

Ramos, M. H., van Andel, S. J., and Pappenberger, F.: Do probabilistic forecasts lead to better decisions?, Hydrol. Earth Syst. Sci., 17, 2219-2232, doi:10.5194/hess-17-2219-2013, 2013.

R code software: available at: http://www4.stat.ncsu.edu/ bondell/ software.html (last access: 1 October 2013), NC State 20 University Department of Statistics (NCSU Statistics), North Carolina, USA, 2010.

R Core Team: R: a Language and Environment for Statistical Computing, available at: http://www.R-project.org/, R Foundation for Statistical Computing, Vienna, Austria, 2013.

Reggiani, P., Renner, M., Weerts, A., and Van Gelder, P.: Uncertainty assessment via Bayesian revision of ensemble streamflow predictions in the operational river Rhine forecasting system, Water Resour. Res., 45, W02428, doi:10.1029/2007WR006758, 2009.

Regonda, S. K., Seo, D.-J., Lawrence, B., Brown, J. D., and Demargne, J.: Short-term ensemble streamflow forecasting using operationally-produced single-valued streamflow forecasts - A Hydrologic Model Output Statistics (HMOS) approach, J. Hydrol., 497, 80-96, 2013.

Roscoe, K. L., Weerts, A. H., and Schroevers, M.: Estimation of the uncertainty in water level forecasts at ungauged river locations using Quantile Regression, Int. J. River Basin Manage., 10, 383 394, 2012.

Schellekens, J., Weerts, A. H., Moore, R. J., Pierce, C. E., and Hildon, S.: The use of MOGREPS ensemble rainfall forecasts in operational flood forecasting systems across England and Wales, Adv. Geosci., 29, 77-84, doi:10.5194/adgeo-29-77-2011, 2011.

Sene, K., Weerts, A., Beven, K., Moore, R., Whitlow, C., and Young, P.: Risk-based probabilistic fluvial flood forecasting for integrated catchment models? Phase 1 Report, Science Report SC080030/SR1, Joint Defra / Environment Agency Flood and Coastal Erosion Risk Management Research and Development Programme, Rio House, Waterside Drive, Aztec West, Almondsbury, Bristol, BS32 4UD, available at: http://evidence. environment-agency.gov.uk/FCERM, 2009.
Seo, D.-J., Herr, H. D., and Schaake, J. C.: A statistical postprocessor for accounting of hydrologic uncertainty in short-range ensemble streamflow prediction, Hydrol. Earth Syst. Sci. Discuss., 3, 1987-2035, doi:10.5194/hessd-3-1987-2006, 2006.

Smith, P. J., Panziera, L., and Beven, K. J.: Forecasting flash floods using data-based mechanistic models and NORA radar rainfall forecasts, Hydrol. Sci. J., 1-15, 1403-1417, doi:10.1080/02626667.2013.842647, 2014.

Solomatine, D. P. and Shrestha, D. L.: A novel method to estimate model uncertainty using machine learning techniques, Water Resour. Res., 45, W00B11, doi:10.1029/2008WR006839, 2009.

Todini, E.: A model conditional processor to assess predictive uncertainty in flood forecasting, Int. J. River Basin Manage., 6, 123-137, 2008.

van Andel, S. J., Weerts, A., Schaake, J., and Bogner, K.: Post-processing hydrological ensemble predictions intercomparison experiment, Hydrol. Process., 27, 158-161, doi:10.1002/hyp.9595, 2013.

Van Steenbergen, N., Ronsyn, J., and Willems, P.: A non-parametric data-based approach for probabilistic flood forecasting in support of uncertainty communication, Environ. Modell. Softw., 33, 92 105, 2012.

Vaughan, M.: Probabilistic flood forecasting (Environment Agency), 85th European Study Group with Industry, 1620 April 2012, University of East Anglia, Norwich, 2012.

Verkade, J. S. and Werner, M. G. F.: Estimating the benefits of single value and probability forecasting for flood warning, Hydrol. Earth Syst. Sci., 15, 3751-3765, doi:10.5194/hess-15-37512011, 2011.

Verkade, J. S., Brown, J., Reggiani, P., and Weerts, A.: Post-processing ECMWF precipitation and temperature ensemble reforecasts for operational hydrologic forecasting at various spatial scales, J. Hydrol., 501, 73-91, doi:10.1016/j.jhydrol.2013.07.039, 2013.

Wallingford: Wallingford Water, a flood forecasting and warning system for the river Soar, Wallingford Water, Wallingford, UK, 1994.

Wallingford: HR Wallingford, ISIS software, available at: http://www.isisuser.com/isis/ (last access: 1 October 2013), HR Wallingford, Hydraluic Unit, Wallingford, UK, $1997 .$.

Weerts, A. H., Winsemius, H. C., and Verkade, J. S.: Estimation of predictive hydrological uncertainty using quantile regression: examples from the National Flood Forecasting System (England and Wales), Hydrol. Earth Syst. Sci., 15, 255-265, doi:10.5194/hess-15-255-2011, 2011.

Werner, M., Schellekens, J., Gijsbers, P., van Dijk, M., van den Akker, O., and Heynert, K.: The Delft-FEWS flow forecasting system, Environ. Modell. Softw., 40, 65-77, doi:10.1016/j.envsoft.2012.07.010, 2013.

Wilks, D.: Statistical Methods in the Atmospheric Sciences, Academic Press, San Diego, California, USA, 2006. 